\title{
Evaluación de la dinámica de población de bacterias magnetotácticas (MTBs) en medios naturales y enriquecidos, comparación con resultados teóricos obtenidos a partir del modelo de competencia de Lotka-Volterra
}

\author{
Alejandro Salazar Villegas ${ }^{1} \mid$ Viviana Morillo López ${ }^{1} \mid$ Álvaro Morales Aramburo ${ }^{2}$ | Marco Márquez Godoy ${ }^{3}$
}

Recibido:

14 de abril de 2009

Aceptado:

10 de junio de 2009

\section{Resumen}

Todas las MTBs reportadas hasta la fecha, han compartido la propiedad de ser muy difíciles de cultivar. Sin embargo, las características particulares de los nanocristales magnéticos (comúnmente de magnetita o greigita) que crecen en el interior de estos microorganismos, hace que sea muy interesante la búsqueda de estrategias que permitan obtener grandes cantidades de estas partículas. En este trabajo se presenta un método para aumentar la tasa de crecimiento de MTBs provenientes del embalse de La Fe, Colombia, enriqueciendo su medio natural con una solución de vitaminas, una solución de minerales y quinato férrico como fuente de hierro. Además, se utiliza el modelo de competencia de Lotka-Volterra para identificar posibles factores que sean determinantes en la interacción de las MTBs con su entorno biótico. Para esto se consideran las MTBs como una especie y los microorganismos no-magnetotácticos como la especie rival. Los resultados sugieren una curva de crecimiento con dos etapas diferenciables. Una etapa inicial que puede considerarse como la fase lag, toma alrededor de 25 días, y una etapa de crecimiento poblacional (fase Log) con un máximo en el día 60. Es posible que esta técnica de enriquecimiento aumente hasta 6.1 veces la población de MTBs naturales. El nivel de correlación entre los resultados teóricos y los experimentales es significativo. Esto sugiere que hay factores de competencia entre las MTBs y los microorganismos no-magnetotácticos de su ambiente natural, que influyen de manera importante en su dinámica de población. Se requieren otros estudios para confirmar esta hipótesis.

Palabras Clave: Bacteria magnetotáctica (MTB); dinámica de población; enriquecimiento; cultivo de bacterias.

${ }^{1}$ Facultad de Ciencias, Universidad Nacional de Colombia, Sede Medellín. asalazar1370@gmail.com, vivikamorillo@gmail.com. 2Instituto de Física, Universidad de Antioquia, A.A. 1226, Medellín, Colombia. amoral@fisica.udea.edu.co ${ }^{3}$ Facultad de Minas, Universidad Nacional de Colombia, Sede Medellín. Grupo de Mineralogía Aplicada y Bioprocesos mmarquez@unalmed.edu.co

\section{Abstract}

All MTB reported to date share the characteristic of being very difficult to cultivate. Nevertheless, the particular features of the magnetic nanocrystals (commonly of magnetite or gregite) that grow inside these microorganisms, makes very interesting the search for strategies to obtain large quantities of these 
particles. In this paper a method is presented to increase the growth rate of MTB from the reservoir of $\mathrm{La} \mathrm{Fe}$, Colombia, enriching its natural environment with a vitamin solution, a mineral solution and ferric quinate as iron source. In addition, the competitive Lotka-Volterra model was used to identify possible factors that could be important in the interaction between MTB and their biotic surrounding. For this, the MTBs were considered as one species and all the non magnetotactic microorganisms as the rival species. The results suggest a growth curve with two differentiable stages. An initial stage that can be considered as the lag phase, takes about 25 days, and a population growth phase (log phase) with its maximum on day 60. The MTB population of one of the enriched samples increased up to 6.1 times its original size. The level of correlation between experimental and theoretical results is significant. This suggests that there are competitive factors between MTB and the non magnetotactic microorganisms of its natural environment, which have a strong influence in its population dynamics. Further studies are required to confirm this hypothesis.

Keywords: Magnetotactic bacteria (MTB); Population dynamic; Enrichment; Competitive factors; Bacteria culture

\section{Introducción}

L as bacterias magnetotácticas son microorganismos acuáticos, procariotas, Gram negativos que tienen la propiedad de orientar sus movimientos en función del campo magnético del ambiente en el que se encuentren [1]. Esta propiedad se conoce como magnetotáxis [2] y se debe principalmente a la presencia de nanocristales magnéticos (los más comunes siendo magnetita y greigita) $[3,4]$, recubiertos por una bicapa lipídica, que están organizados en el interior de la célula en cadenas que se orientan con las líneas del campo magnético [2]. Estas cadenas se conocen como magnetosomas [2]. Se han encontrado muchos tipos de MTBs en diferentes ambientes acuáticos [5,6,7]. Entre los reportados hay cocos, espirilos, bacilos, vibrios y agregados multicelulares $[5,6,7]$.

Utilizando este mecanismo de orientación, las bacterias se ubican en regiones específicas de los gradientes químicos [5,8]. Sin embargo, aún no se ha logrado correlacionar de manera precisa su posición en los diferentes gradientes, con parámetros geoquímicos [9].

En este trabajo se espera obtener resultados que permitan determinar si el enriquecimiento de medios naturales induce un aumento en la población de MTBs nativas o no. Además, se hace un seguimiento del crecimiento de las poblaciones de MTBs, en muestras enriquecidas y no enriquecidas, con el ánimo de obtener luces que permitan predecir la dinámica de la población de estas bacterias. Por último, se comparan las curvas de crecimiento obtenidas experimentalmente, con otra generada teóricamente, a partir del modelo de competencia de Lotka-Volterra. Éste es un modelo muy general, deducido de manera independiente por Lotka (1925) y Volterra (1926), que le permite a diferentes especies interactuar a través de un acoplamiento nolineal [10]. Los parámetros utilizados en este procedimiento fueron supuestos con base en observaciones hechas en el laboratorio. Los modelos de Lotka-Volterra han sido utilizados ampliamente en el estudio de la dinámica de especies interactuantes y en otros campos [11], como la modelación económica. 


\section{Materiales y métodos}

\section{Zona de muestreo:}

Todas las muestras fueron tomadas en la represa de la $\mathrm{Fe}$, ubicada en el municipio del Retiro (6o 6' $57^{\prime \prime}$ norte a 6o 6' $00^{\prime \prime}$ norte y 75을 $30^{\prime} 30^{\prime \prime}$ oeste a $75^{\circ} 29^{\prime} 36^{\prime \prime}$ oeste) a una altitud de 2156 m.s.n.m.

\section{Toma de muestras:}

Las muestras de agua-sedimento fueron tomadas en la zona de transferencia óxicoanóxica (ZTOA), en puntos ubicados al norte de la represa. Para localizar esta región en la columna de agua, se utilizó un oxímetro (HANNA). Las muestras fueron almacenadas en recipientes de volúmenes diferentes, grandes (11l-metodología I) y pequeños (600ml-metodologóa II).

\section{Almacenamiento y enriquecimiento:}

Las muestras se almacenaron a temperatura ambiente y oscuridad total, durante todo el periodo de seguimiento (60 días). El día del muestreo se enriquecieron una muestra grande $(\mathrm{Ge})$ y 12 pequeñas $(\mathrm{Pe})$. También se le hizo seguimiento a una muestra grande y 12 pequeñas no enriquecidas (Gne y Pne respectivamente). El enriquecimiento se hizo con una solución de vitaminas, una solución de minerales y quinato férrico al $0.01 \mathrm{M}$, como fuente de hierro. Las soluciones de vitaminas y minerales fueron preparadas con base en medios de cultivo líquidos que han sido reportados en la literatura como exitosos para MTBs (medio de cultivo de Wolfe. ATCC: 1653 medio de crecimiento de Spirillum magnético (MSGM)). El quinato férrico fue preparado combinando $0.279 \mathrm{~g}$ de $\mathrm{FeCl} 3$ y $0.16 \mathrm{~g}$ de ácido quínico (sigma) en $100 \mathrm{ml}$ de agua destilada. Ambas soluciones fueron previamente autoclavadas antes del enriquecimiento.

\section{Aislamiento y conteo de MTBs:}

Las MTBs fueron aisladas según la técnica de separación descrita por Wajnberg et al. (1986) [12]. Para eliminar las impurezas, las MTBs aisladas fueron concentradas en la pared de un eppendorf, utilizando un pequeño imán de neodimio. Las bacterias separadas de esta manera, se fijaron con una solución de pformaldehido 4\%. El conteo se hizo utilizando cámara de Neubauer [13]. La solución final de la que se tomaron las MTBs en cada conteo estaba compuesta por $800 \mu \mathrm{l}$ de agua de la zona de muestreo, estéril y filtrada, $100 \mu$ lde p-formaldehido $4 \%$ y $100 \mu l$ de de la solución que contenía las MTBs aisladas y con muy pocas impurezas. Por esto, el factor de dilución en la ecuación que se utiliza para el conteo con cámara de Neubauer [13] es 10.

Seguimiento de la dinámica de población de MTBs:

Se hizo conteo de MTBs cada 5 días siguiendo dos metodologías.

Metodología I: Se almacenaron muestras en dos recipientes de 11 litros. Una de las muestras fue enriquecida como se explicó anteriormente. Se produjo una curva de crecimiento con los datos obtenidos de los conteos de MTBs contenidas en cada uno de los dos recipientes. Esta metodología tiene la ventaja de que se le hace el seguimiento a la misma población de MTBs en todo momento. Tiene como desventaja que en cada conteo se está agotando la población, pues la técnica mata la población de bacterias sometida al procedimiento.

Metodología II: Se almacenaron 24 muestras en recipientes de $600 \mathrm{ml} .12$ de estas muestras fueron enriquecidas como se explicó. Cada 5 días se hizo conteo en una muestra enriquecida y en una no enriquecida, descartando las muestras utilizadas en cada procedimiento. Este método tiene la ventaja de que las muestras utilizadas en cada conteo no han sido alteradas desde el momento del muestreo (no hay agotamiento de MTBs). Sin embargo, tiene la desventaja de que probablemente las 
poblaciones iniciales de MTBs en cada muestra son diferentes, aunque se hayan tomado de la misma zona.

\section{Modelo de competencia de Lotka-Volterra:}

En este modelo, un sistema de $\mathrm{N}$ especies (o agentes) con una población $\mathrm{x}_{\mathrm{i}}$, para $\mathrm{i}=0$ hasta $\mathrm{N}$, que compite por un conjunto de recursos finitos y fijos, se puede describir por la siguiente ecuación [12]:

$$
\frac{d x_{i}}{d t}=r_{i} x_{i}\left(1-\sum_{j=1}^{N} a_{i j} x_{j}\right)
$$

Donde $r_{i}$ representa la tasa de crecimiento lineal de la especie $i, \mathrm{~A}=\left(a_{i j}\right)$ es la matriz de interacción y $a_{i j}$ representa la interacción de la especie $i$ con la especie $j$ (la interacción entre las especies no es necesariamente simétrica, y generalmente $a_{i j} \neq a_{j i}$ ) [10]. Esta ecuación se conoce como ecuación de competencia o de competencia interespecífica de Lotka-Volterra. La versión puramente competitiva de este modelo requiere que todos los $a_{i j} \geq 0$ [10], pues el efecto de la interacción debe afectar negativamente la tasa de crecimiento de la población de la(s) especie(es) con que se compite.

En este trabajo se considera como especie $1\left(x_{1}\right)$ : microorganismos magnetotácticos, y especie $2\left(x_{2}\right)$ : microorganismos nomagnetotácticos. Las ecuaciones de competencia de Lotka-Volterra son entonces:

$$
\begin{aligned}
& \frac{d x_{1}}{d t}=r_{1} x_{1}\left(1-x_{1}-a_{12} x_{2}\right) \\
& \frac{d x_{2}}{d t}=r_{2} x_{2}\left(1-x_{2}-a_{21} x_{1}\right)
\end{aligned}
$$

Los valores de los parámetros y las condiciones iniciales, supuestos con base en observaciones hechas en el laboratorio, son: $r_{1}=1, \quad r_{2}=0.1, \quad a_{12}=0.1, \quad a_{21}=1, \quad x_{1}(0)=0.7$, $\mathrm{x}_{2}(0)=50$. Los valores de $r$ y $a$ se eligieron bajo el supuesto de que las condiciones de oscuridad favorecen el crecimiento de las MTBs. Es decir, el que $a_{12}=\left(0.1 a_{21}\right)$ se debe interpretar como que las condiciones de almacenamiento ejercen un efecto 10 veces más dañino sobre los microorganismos fotosintéticos, que son la gran mayoría de los no-magnetotácticos, que sobre las MTBs. Además, como se puede ver a partir de la ecuación 1, existe una relación inversa de proporcionalidad entre el parámetro $a_{i j}$ y el crecimiento en el tiempo de la población de la especie $i, d x_{i} / d t$.

Análogamente, se asume que las poblaciones del conjunto de microorganismos que conforman la denominada especie 2, crecen a una tasa 10 veces menor de lo que lo hacen las MTBs, que no se afectan por las condiciones de oscuridad $\left(r_{1}=10 r_{2}\right)$. Es decir, el crecimiento en el tiempo de la población de MTBs se da a una velocidad 10 veces mayor que lo que lo hace la población nomagnetotáctica (conformada en su mayoría por microorganismos fotosintéticos), cuando el microcosmos está sometido a condiciones de baja luminosidad.

En este trabajo no se hizo conteo de los microorganismos no-magnetotácticos, es decir, no se tienen valores experimentales de $x_{2}$. No obstante, las observaciones en el laboratorio sugieren que la población natural (inicial) de microorganismos no-magnetotácticos es muy superior a la de las MTBs. Los valores $x_{1}(0)=0.7$ y $x_{2}(0)=50$, se eligieron de manera que la razón $x_{1}(0) / x_{2}(0)$ se aproximara al promedio de las observaciones.

Para solucionar estas ecuaciones, se utilizó el método de Runge-Kutta de cuarto orden, con un tamaño de paso $h=0.001$. 


\section{Resultados}

\section{Dinámica de la población de MTBs:}

En la figura 1, se observa la dinámica de la población de MTBs cuando se utilizó la metodología I para hacer el seguimiento.

Figura 1. Dinámica de la población de MTBs

(Metodología I). En la figura se observa el comportamiento de la población en las muestras enriquecida $(\mathrm{Ge})$ y no enriquecidas (Gne).

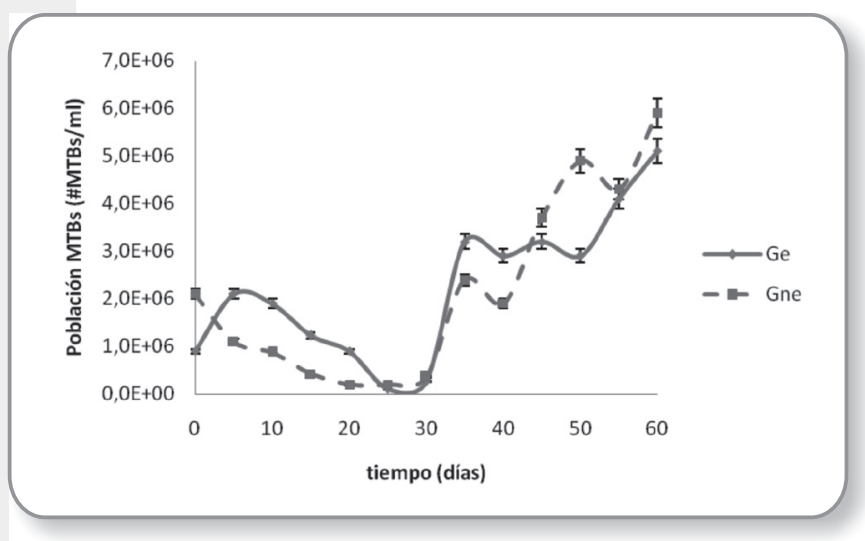

Los resultados obtenidos mediante la metodología II se pueden observar en la figura 2 .

Figura 2. Dinámica de la población de MTBs

(Metodología II). En la figura se observa el comportamiento de la población en las muestras enriquecida $(\mathrm{Pe})$ y no-enriquecidas (Pne).

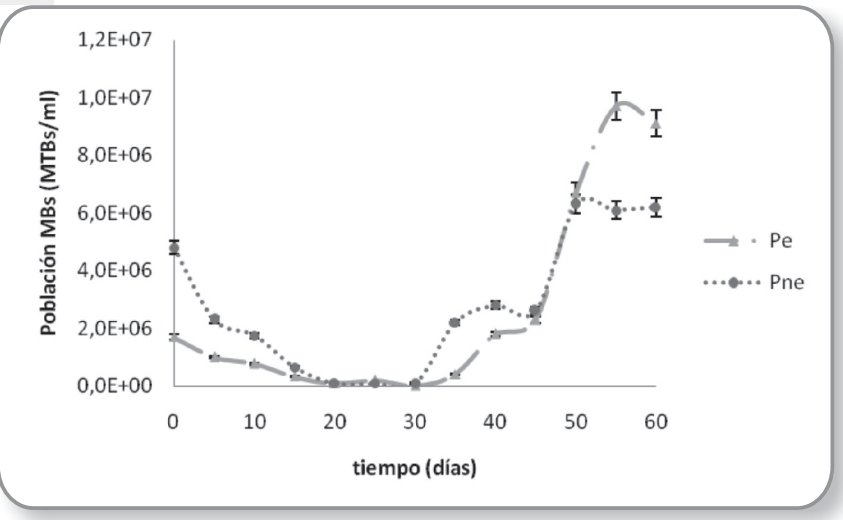

En las figuras $3 \mathrm{a}$ y $3 \mathrm{~b}$ se observa la comparación de los métodos I y II, utilizados para hacer el seguimiento a las poblaciones de MTBs.
Figura 3a. Dinámica de la población de MTBs. Muestras enriquecidas. Las curvas fueron generadas a partir de datos tomados de muestras grandes (metodología I) y pequeñas (metodología II).

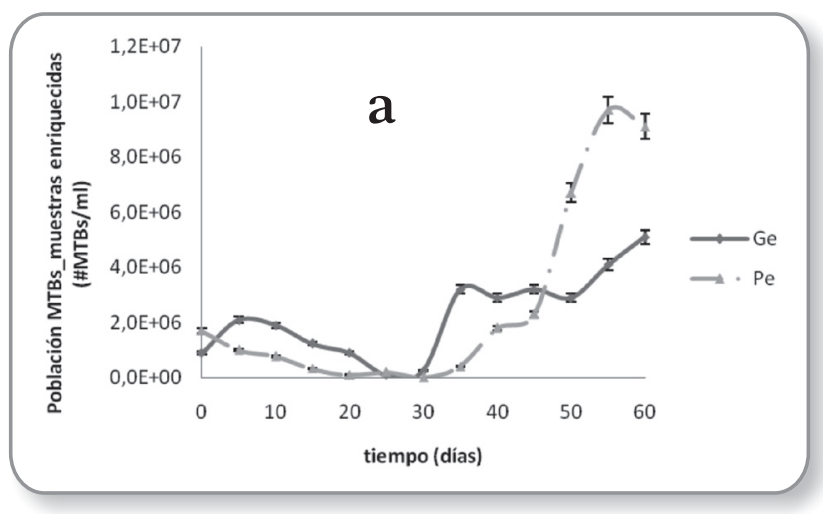

Figura 3b. Dinámica de la población de MTBs. Muestras no-enriquecidas. Las curvas generadas a partir de datos tomados de muestras grandes (metodología I) y pequeñas (metodología II).

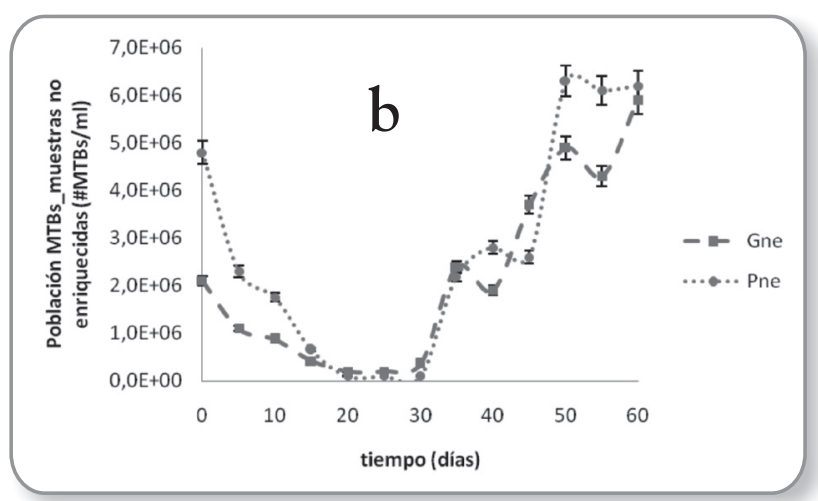

Por último, se muestra el comportamiento de las poblaciones de MTBs en los cuatro casos (figura 4).

Figura 4. Dinámica de la población de MTBs. En la figura se observa el comportamiento de las poblaciones en las muestras enriquecidas y no enriquecidas (utilizando ambas metodologías).

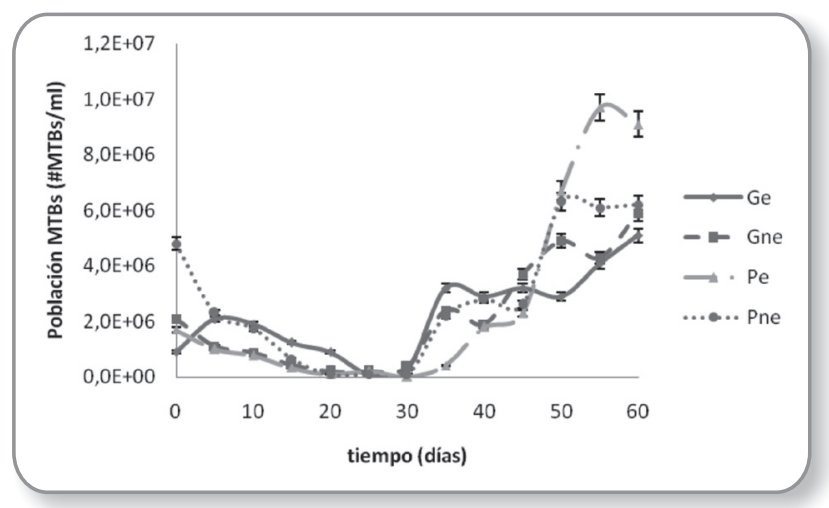


En esta figura se ve de manera más clara que las poblaciones de mayor tamaño, registradas en los últimos conteos, corresponden a las muestras pequeñas, es decir a la metodología II.

\section{Resultados experimentales vs. Modelo teórico:}

El modelo de competencia de LotkaVolterra, para los valores de los parámetros y las condiciones iniciales supuestas, sugiere que la dinámica de población de las dos especies consideradas es de la siguiente forma (figura 5):

Figura 5. Dinámica de las poblaciones de dos especies según el modelo de competencia de Lotka-Volterra, cuando las condiciones son: $\mathrm{r}_{1}=1, \mathrm{r}_{2}=0.1, \mathrm{a}_{12}=0.1$, $\mathrm{a}_{21}=1, \mathrm{x}_{1}(0)=0.7, \mathrm{x}_{2}(0)=50$. En la figura la línea continua representa la población de la especie 1 (magnetotáctica) y la discontinua la de la especie 2 (no-magnetotáctica).

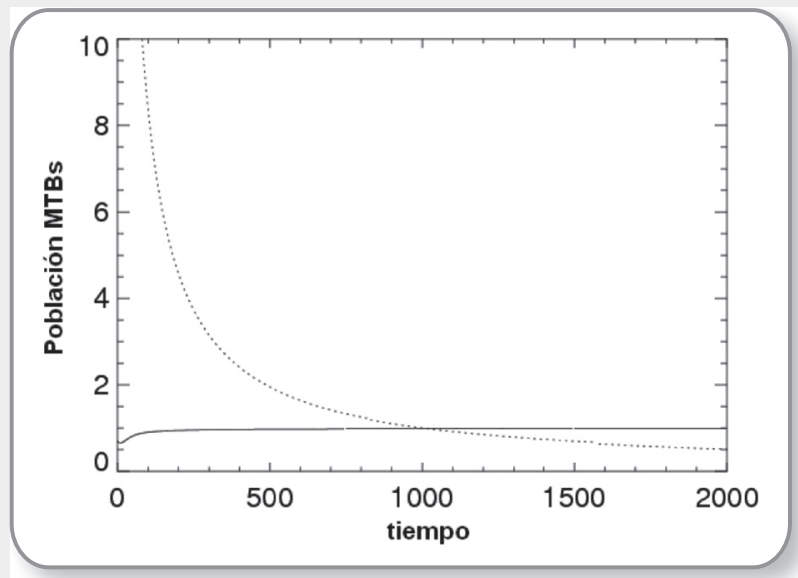

En la figura 6 se hace un acercamiento a la figura 5, de manera que se observe con más detalle el comportamiento de la población de la especie 1 al inicio del experimento.

Figura 6. Dinámica de las poblaciones de dos especies según el modelo de competencia de Lotka-Volterra, cuando las condiciones son: $\mathrm{r}_{1}=1, \mathrm{r}_{2}=0.1, \mathrm{a}_{12}=0.1$, $\mathrm{a}_{21}=1, \mathrm{x}_{1}(0)=0.7, \mathrm{x}_{2}(0)=50$. Esta es una región aumentada de la figura 5 , en la que se ve con más detalle la dinámica de la población de la especie

1 (MTBs) al inicio del experimento. El eje de las ordenadas está normalizado con los ejes de las figuras 7a, b, c y d.

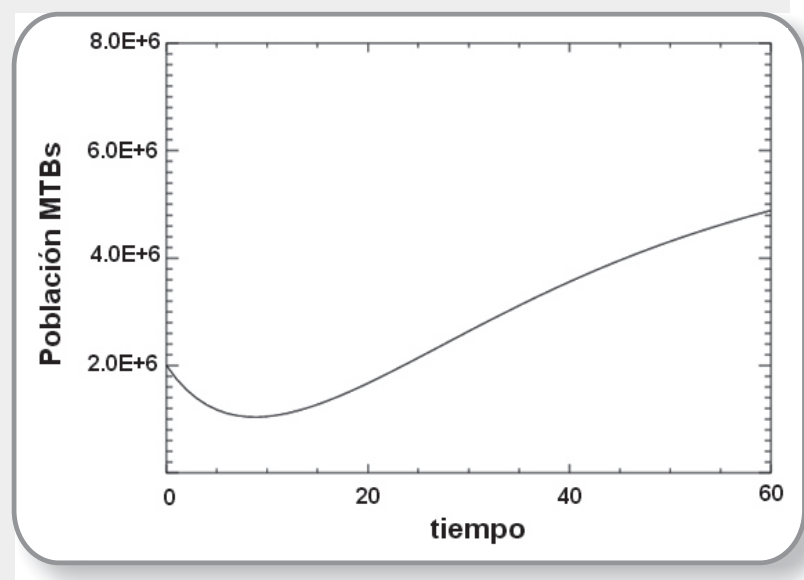

A continuación, se muestran las gráficas que contienen las líneas de tendencia que promedian el comportamiento de la población de MTBs para cada caso experimental.

Figura 7. Dinámica de la población de MTBs. Datos experimentales. Se hace una línea de tendencia en cada caso, de manera que se puedan comparar estos resultados con la curva obtenida del modelo de Lotka-Volterra. Muestras (a) Grande enriquecida (Ge), (b) Grande no-enriquecida (Gne), (c) Pequeñas enriquecidas (Pe) y (d) Pequeña noenriquecida (Pne).
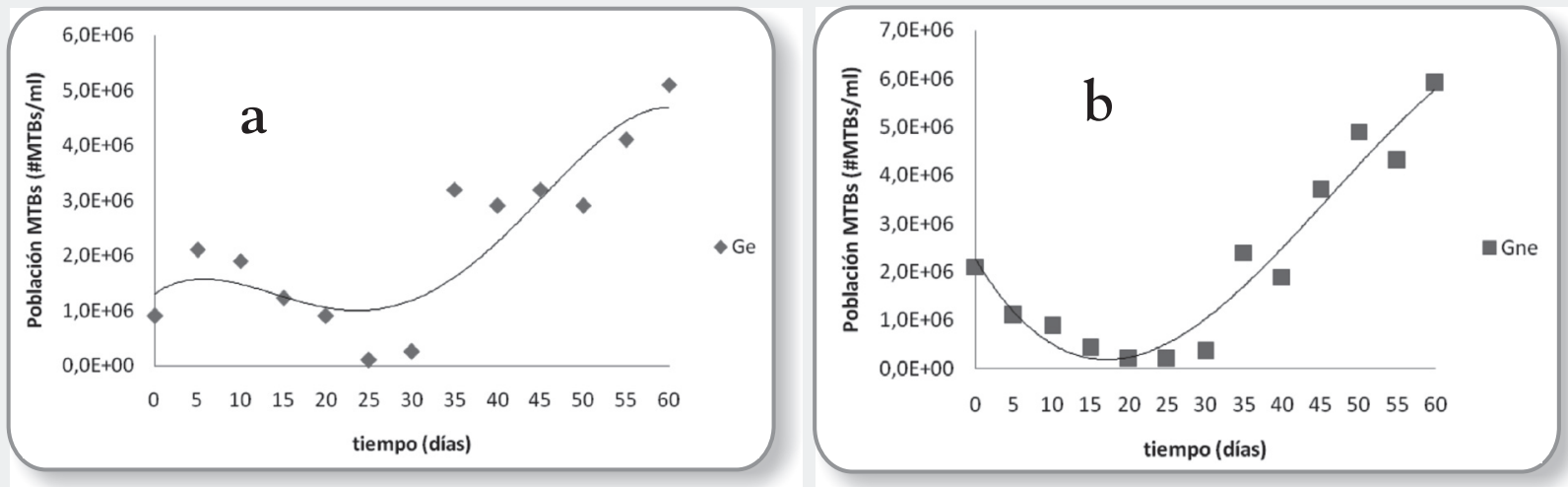


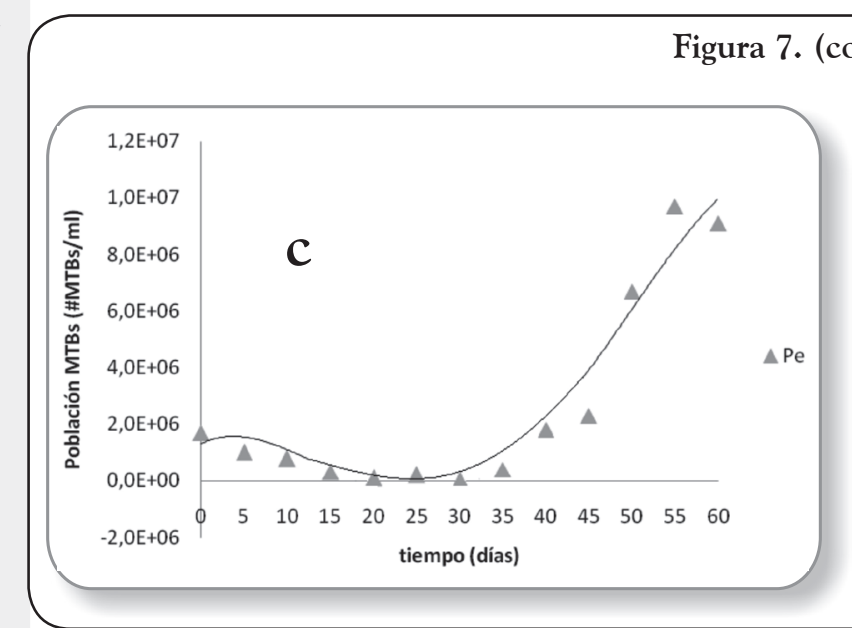

comparación con resultados teóricos obtenidos a partir del modelo de competencia de Lotka-Volterra

ISSN 0122-820X

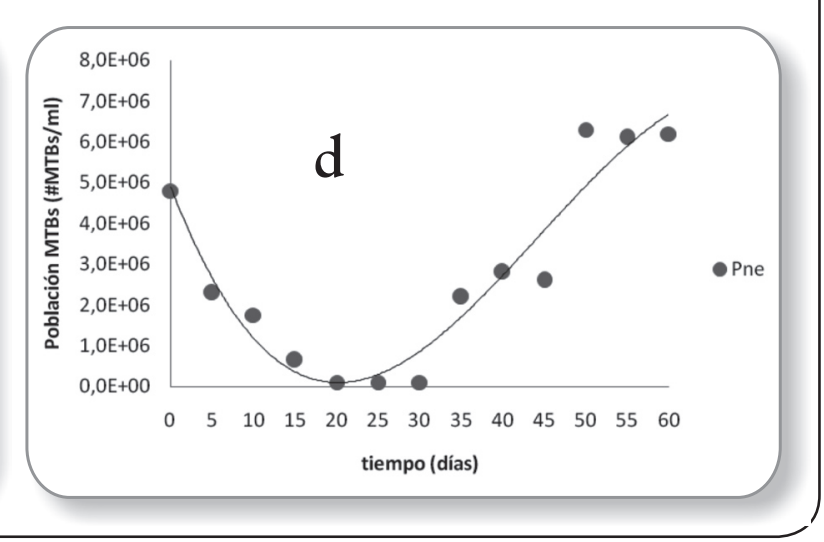

Otro análisis interesante es de cómo varían las poblaciones, una en función de la otra. El espacio de fases que se genera en este caso es el que se muestra en la figura 8:

Hay que resaltar que en la figura 8 , al igual que en la 5, la especie 2 (no-magnetotáctica) no se extingue. Su población decrece asintóticamente pero no llega a ser cero. Además, en esta figura se distinguen tres etapas que representan momentos diferentes del proceso que se intenta modelar. Etapa I: Las poblaciones de ambas especies decrecen. Etapa II: La población de la especie 2 decrece y la de la especie 1 crece. La tasa de crecimiento de población de la especie 1 aumenta con el tiempo. Etapa III: La población de la especie 2 decrece y la de la especie 1 crece. La tasa de crecimiento de población de la especie 1 disminuye con el tiempo.

Figura 8. Espacio de fases en el que se indica la dinámica de la población de una especie en función del tamaño de población de la otra.

\section{Discusión}

Todas las curvas obtenidas experimentalmente, aunque diferentes entre sí, tienen un mismo patrón en el que se pueden diferenciar por lo menos dos etapas. En la etapa inicial, que dura entre 20 y 30 días, la población de MTBs se reduce drásticamente, a una razón casi constante. En la segunda etapa, que le sigue, la población aumenta a una tasa similar a la que se disminuyó en la primera etapa, hasta lo que parece un valor máximo de las poblaciones, cerca al día 60 (figura 4).

La figura 1, sugiere que el proceso de enriquecimiento, por lo menos con los reactivos utilizados en este estudio y durante el periodo en el que se hizo el seguimiento, no favorece ni desfavorece de manera significativa el crecimiento de la población de MTBs provenientes del embalse de $\mathrm{La} \mathrm{Fe}$, a lo sumo, puede ser el responsable de un ligero aumento de la población de MTBs entre los días 5 y 20. Este es un resultado más que confirma la dificultad que representa obtener grandes cantidades de MTBs por medio de cultivo o enriquecimiento de muestras naturales $[2,14,15,16]$.

En la figura 2, se observa el mismo comportamiento de las poblaciones de MTBs que en la figura 1. No obstante, en este caso la comparación entre los tamaños de población al inicio y al final del experimento sugiere que la técnica de enriquecimiento 
indujo significativamente el crecimiento de la población de MTBs (tabla 1).

Tabla 1. Cambio en la concentración de MTBs en muestras enriquecidas (Pe) y no enriquecidas (Pne). Caso metodología II.

\begin{tabular}{|c|c|c|c|}
\hline Muestra & $\begin{array}{c}\text { Concentració } \\
\mathbf{n} \text { inicial } \\
(\mathbf{M T B s} / \mathbf{m l})\end{array}$ & $\begin{array}{c}\text { Concentración } \\
\text { final (MTBs/ml) }\end{array}$ & $\begin{array}{c}\text { Aumento de } \\
\text { concentració } \\
\mathbf{n}(\mathbf{M T B s} / \mathbf{m l})\end{array}$ \\
\hline $\mathrm{Pe}$ & $1.6 \times 10^{6}$ & $9.6 \times 10^{6}$ & $8.0 \times 10^{6}$ \\
\hline Pne & $4.7 \times 10^{6}$ & $6.1 \times 10^{6}$ & $1.4 \times 10^{6}$ \\
\hline
\end{tabular}

Esto significa que existe la posibilidad de que, por medio de técnicas de enriquecimiento, se induzca un crecimiento de hasta 6.1 veces en las poblaciones naturales de MTBs, en un periodo de 60 días. Futuros estudios podrían no sólo confirmar esta hipótesis, sino también esclarecer los requerimientos nutricionales de estos microorganismos tan exigentes.

De la figura $3 b$, se puede inferir que cualquiera de las metodologías utilizadas para hacer el seguimiento es válida o, por lo menos, que arrojan resultados muy similares. No obstante, las figuras 3a y 4 parecen indicar que el constante agotamiento de la población de MTBs, practicado en la metodología I, puede disminuir de manera significativa su tamaño. De ser así, el método generaría una curva de crecimiento que no representa la dinámica de la población de MTBs en condiciones naturales. Sin embargo, no se descarta la posibilidad de que haya un factor no detectado, como un suelo ligeramente más nutritivo o una ecología más favorable, que induzca la diferencia entre de las curvas que se observan en la figura $3 a$ y 4 . Por estas razones, la sugerencia es que se utilicen ambos métodos para minimizar los posibles errores que puedan surgir en el proceso de conteo.

La dinámica de las poblaciones observada en la figura 5 se correlaciona de manera muy interesante a las observaciones realizadas en el laboratorio, no sólo en el desarrollo de este trabajo sino a lo largo de toda la investigación sobre MTBs. Aunque no se ha hecho un seguimiento cuantitativo de los microorganismos no magnetotácticos que hacen parte del microambiente que contiene las MTBs, siempre se ha registrado una disminución drástica de dichas poblaciones, debido a que las muestras son almacenadas en condiciones de oscuridad y la gran mayoría de estos microorganismos no magnetotácticos son fotosintéticos. Sólo en casos aislados una especie no-magnetotáctica y no-fotosintética ha proliferado sola o junto a las MTBs.

El objetivo de las figuras 6 y 7 es señalar la similitud entre el modelo obtenido de manera teórica con las ecuaciones de competencia de Lotka-Volterra y las líneas de tendencia generadas a partir de los valores de tamaño de población obtenidos de manera experimental. Vale la pena resaltar que las líneas de tendencia en los casos en que la muestra fue previamente enriquecida (Figuras $7 \mathrm{a}$ y $7 \mathrm{c}$ ), indican un ligero aumento de la población de MTBs en la primera etapa de la curva de crecimiento, mientras que las poblaciones de las muestras no-enriquecidas (Figuras $7 \mathrm{~b}$ y $7 \mathrm{~d}$ ) muestran un decrecimiento de la población de MTBs muy similar al que experimenta la especie 1 (MTBs) en el modelo de Lotka-Volterra (Figura 6).

Las etapas de la figura 8 pueden interpretarse de la siguiente manera. Etapa I: ambas especies son desfavorecidas por las condiciones del sistema que las contiene. El impacto negativo parece ser proporcional al tamaño de la población de cada especie. Esto sugiere que el factor que desfavorece el crecimiento de ambas especies es la diferencia tan grande entre los tamaños de sus poblaciones en el momento inicial. Etapa II: La población de la especie 1 alcanza un mínimo después del cual las condiciones del sistema favorecen su crecimiento. El sistema puede considerarse lejos del equilibrio, pues la tasa de crecimiento de la población de la especie 1 respecto a la especie 2 aumenta en el tiempo. Etapa III: El sistema se acerca al equilibrio (sin que signifique que tenga que llegar a él en algún momento) por lo que la tasa de crecimiento de la población de la especie 1 decrece en el tiempo. La comparación de las figuras 8 y 5 permite visualizar que el tiempo 
que duran las etapas I y II es muy pequeño respecto al gran espacio de tiempo que el sistema permanece en la etapa III. Se debe tener en cuenta que todo análisis que se haga sobre la figura 8 es meramente especulativo, pues no se ha hecho un seguimiento real de las poblaciones de microorganismos no magnetotácticos (especie 2). Sin embargo, ya que la figura 8 está estrechamente relacionada con la figura 5 y esta última se acerca mucho al comportamiento de las poblaciones deducido de observaciones y mediciones, su análisis no puede ser menos que interesante.

\section{Conclusiones}

La curva de crecimiento poblacional de las MTBs de la represa La Fe, puede dividirse en dos etapas que representan fases diferentes en la dinámica de esta población. En la primera, la población decrece drásticamente hasta alcanzar un valor mínimo, cerca al día 30. En la segunda la población crece hasta un máximo que depende de factores ambientales y de características inherentes a cada especie. Esta última etapa también tiene una duración aproximada de 30 días.

Losresultadossugierendoshechosconcretos respecto a la técnica de enriquecimiento. El primero es que ninguno de los compuestos adicionados a los medios naturales inhibe el crecimiento de las MTBs ni la síntesis de magnetosomas. El segundo es la posibilidad de que, dependiendo de las condiciones iniciales de las muestras (composición, población inicial de MTBs, volumen del recipiente que las contiene) y de las condiciones de almacenamiento (luminosidad, temperatura, reposo), esta técnica de enriquecimiento puede inducir el crecimiento de poblaciones de MTBs hasta 6.1 veces su tamaño inicial, en un periodo de dos meses.

Las dos metodologías utilizadas para hacer el seguimiento de las poblaciones de MTBs son adecuadas, no obstante, la utilización simultánea de ambas técnicas, disminuye significativamente los posibles errores que puedan acumularse en los conteos.

El modelode competenciade Lotka-Volterra genera curvas que se ajustan de manera significativa a los resultados experimentales obtenidos en el estudio de la dinámica de población de las MTBs de la represa La Fe. No obstante, dada la simpleza del modelo y la complejidad del sistema objeto de estudio, no es posible determinar si entre las MTBs y los microorganismos no-magnetotácticos que hacen parte de su ecología hay o no una estricta relación de competencia del tipo que sugieren los modelos de Lotka-Volterra. Sin embargo, el nivel de correlación entre los resultados teóricos y los experimentales sugiere que los factores evaluados en el modelo pueden ser determinantes en la interacción entre las MTBs y sus vecinos no-magnetotácticos. El tamaño inicial de población (x) y la tasa de crecimiento lineal de cada especie (r) aparecen como factores gobernantes en el modelo matemático y por ende candidatos a ser factores clave en la dinámica poblacional de las MTBs de la represa La Fe.

La continuidad de este tipo de estudios facilitará las herramientas que permitan manipular el entrono de las MTBs de manera que se alcancen tamaños máximos de población en periodos de cultivo cortos.

\section{Agradecimientos}

Los autores agradecen al Programa Nacional de Biotecnología de Colciencias por la financiación de este proyecto. A Empresas Públicas de Medellín (EEPPM) y Comfamiliar Comfenalco por autorizar el muestreo y facilitar la logística en la represa La Fe. Al laboratorio de Biomineralogía de la Universidad Nacional, Sede Medellín y a todos quienes apoyan este trabajo. ASV agradece al profesor Juan F. Salazar por su valiosa colaboración en el desarrollo del modelo de Lotka-Volterra. 


\section{Bibliografía}

[1] Blakemore, R. P. Magnetotactic bacteria. (1975) Science 190:377-379.

[2] Shüler D., (2002) Int. Microbiol 5:209.

[3] Bazylinski D. A. (1995) ASM News. 61:337.

[4] Bazylinski D. A. and Moskowitz. (1997)

[5] Blakemore R. P. (1975) Magnetotactic bacteria. Science 190 (4212), 377-379.

[6] Cox L., Popa R., Bazylinsky D. A., Lanoil B., Douglas S., Belz A., Engler D. and Nealson K. H. (2002) Organization and elemental analysis of $\mathrm{P}_{-}, \mathrm{S}_{-}$, and Fe-rich inclusions in a population of freshwater magnetococci. Geomicrobiol. J. 19:387406.

[7] Moench T. T. and Konetzka W. A. (1978) A novel method for the isolation and study of a magnetotactic bacterium. Arch. Microbiol. 119:203-212.

[8] Frankel R.B., Bazylinski D.A., Johnson M.S. and Taylo, B.L. (1997) Magnetoaerotaxis in marine coccoid bacteria. Biophys. J. 73 (2), 994-1000.

[9] Flies C. B., Jonkers H. M., Dirk de Beer, Bosselmann K., Böttcher M. B., Shüler D. (2005) Diversity and vertical distribution of magnetotactic bacteria along chemical gradients in fresh water microcosms. FEMS Microbiology Ecology 52:185-195. doi:10.1016/ j.femsec.2004.11.006.
[10] Wildenberg J. C., Vano J. A. and Sprott J. C. (2005) Complex spatiotemporal dynamics in Lotka-Volterra ring systems. doi:10.1016/j.ecocom.2005.12.001.

[11] Sprott J. C., Wildenberg J. C. and Azizi Y. (2005) A simple spatiotemporal chaotic Lotka-Volterra model. doi:10.1016/ j.chaos.2005.02.015

[12] Wajnberg E., Salvo de Souza L., Lins de Barros H. and Esquivel D. (1986) Astudy of magnetic properties of magnetotactic bacteria. Biophys. J. $451-455$.

[13] http://www.uni-greifswald.de immuteach/ methods/counting_chamber/counting_ chamber.html, consultado marzo de 2009

[14] Flies C. B., Peplies J. and Shüler D. (2005) Combined approach for characterization of uncultivated magnetotactic bacteria from various aquatic environments. doi: $10.1128 /$ AEM.71.5.27232731.2005

[15] Silvera T. S., Martins J. L., Silva K. T., Abreu F. and Lins U. (2007) Microscopy studies on uncultivated magnetotactic bacteria. Modern Research and Educational Topics in Microscopy. 111121.

[16] Liu Y., Gao M., Dai S., Peng K. and Jai R. (2005) Characterization ofmagnetotactic bacteria and their magnetosomes isolated from Tieshan iron ore in Hubei Province of China. 26:597-601. doi:10.1016/ j.msec.2005.07.023. 